\title{
EEL spectroscopic tomography: towards a new dimension in
}

\section{nanomaterials analysis}

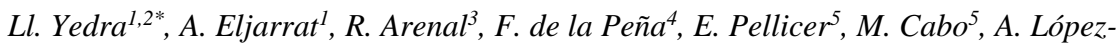

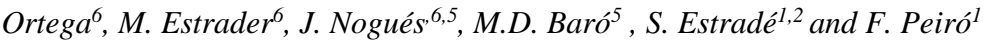

${ }^{1}$ Laboratory of Electron Nanoscopies (LENS)- MIND/IN2UB, Dept. d'Electrònica, Universitat de Barcelona, c/ Martí Franqués 1, E-08028 Barcelona, Spain.

${ }^{2}$ CCiT, Scientific and Technical Center, Universitat de Barcelona, 08028, Barcelona, Spain.

${ }^{3}$ Instituto de Nanociencias de Aragón, c/Mariano Esquillor, ed. I+D, E-50018 Zaragoza, Spain.

4 Department of Materials Science and Metallurgy, University of Cambridge, Pembroke Street, Cambridge CB2 3QZ, United Kingdom.

${ }^{5}$ Departament de Física, Facultat de Ciències, Universitat Autònoma de Barcelona, E-08193 Barcelona, Spain.

${ }^{6} \mathrm{CIN} 2$ (CIN-CSIC) and Universitat Autònoma de Barcelona, Catalan Institute of Nanotechnology, Campus de la UAB, E-08193 Barcelona, Spain.

${ }^{7}$ Institució Catalana de Recerca i Estudis Avançats (ICREA), Campus Universitat Autònoma de Barcelona, E-08193 Barcelona, Spain.

e-mail: llyedra@el.ub.es

\begin{abstract}
:
Electron tomography is a widely spread technique for recovering the three dimensional (3D) shape of nanostructured materials. Using a spectroscopic signal to achieve a reconstruction adds a fourth chemical dimension to the $3 \mathrm{D}$ structure. Up to date, energy filtering of the images in the transmission electron microscope (EFTEM) is the usual spectroscopic method even if most of the information in the spectrum is lost. Unlike EFTEM tomography, the use of electron energy-loss spectroscopy (EELS)
\end{abstract}


spectrum images (SI) for tomographic reconstruction retains all chemical information, and the possibilities of this new approach still remain to be fully exploited. In this letter we prove the feasibility of EEL spectroscopic tomography at low voltages $(80 \mathrm{kV})$ and short acquisition times from data acquired using an aberration corrected instrument and data treatment by Multivariate Analysis (MVA), applied to $\mathrm{Fe}_{\mathrm{x}} \mathrm{Co}_{(3-\mathrm{x})} \mathrm{O}_{4} @ \mathrm{Co}_{3} \mathrm{O}_{4}$ mesoporous materials. This approach provides a new scope into materials: the recovery of full EELS signal in 3D.

\section{Introduction:}

Despite its low energy resolution, EFTEM has prevailed over EELS-SI [1] for analytical electron tomography because of the lower acquisition times and more favorable conditions for sample stability [2-4]. Only a few images (i.e. energy windows) per tilt angle are acquired in EFTEM, which reduces the time of acquisition as well as the energy dose received by the sample. Conversely, EELS-SI had the hindering of high acquisition times and therefore high energy dose. Many samples are beam-sensitive to an extent that impaired this technique to be applied. However, as the new generations of transmission electron microscopes become widely spread, Cs correctors, monochromated sources and more sensitive detectors have enabled higher spatial and energy resolution as well as a decreasing in acquisition time of images and spectra [5]. Operation of the microscope at low voltage also reduces the energy dose on the samples. If we consider MVA [6,7] of the final data, low signal to noise ratio is acceptable, further reducing acquisition times. And last but not least, the proposed low voltage EELS-SI-tomography technique provides a wide range of signals fulfilling the projection requirement, as core-loss signals $[1,8]$, as well as high-angle annular dark field (HAADF) signal, which have already been used for 3D reconstruction [8-11]. Moreover, mathematical treatment of spectra to identify independent components maps from independent component analysis (ICA) is demonstrated to be a useful procedure to retrieve signals which give complementary information to direct elemental quantification from EELS spectra

\section{Materials and methods}

In this work 3D spectrum image tomography analysis is applied to antiferromagnetic (AFM) mesoporous $\mathrm{Co}_{3} \mathrm{O}_{4}$ nanocast replicas of Si KIT-6 filled with ferrimagnetic (FiM) $\mathrm{Fe}_{\mathrm{x}} \mathrm{Co}_{(3-\mathrm{x})} \mathrm{O}_{4}$. A detailed report about the synthesis, structural and magnetic characterisation of these materials as a function of the different concentrations of the FiM phase used for impregnation has been already published [ref paper eva]. Preliminary tomographic results revealed the sequence of deposition of the $\mathrm{Fe}_{\mathrm{x}} \mathrm{Co}_{(3-\mathrm{x})} \mathrm{O}_{4}$ in the porous wall as the charge of impregnation was increased. Nevertheless, iron saturation was detected for an amount of $\mathrm{Fe}_{\mathrm{x}} \mathrm{Co}_{(3-\mathrm{x})} \mathrm{O}_{4}$ of $2.4 \mathrm{Fe}(\mathrm{III}): \mathrm{Co}_{3} \mathrm{O}_{4}$ molar ratio. Previous EELS studies also showed an increased $\mathrm{Fe}$ content for higher charge. However, 2D EELS was unable to detect the spatial distribution of $\mathrm{Fe}$ in depth, as the signal is integrated along the thickness of the particle. Therefore one of the samples (charge $1.2 \mathrm{Fe}(\mathrm{III}): \mathrm{Co}_{3} \mathrm{O}_{4}$ molar ratio) has been selected to demonstrate low voltage EELS-SI-tomography as a suitable technique to assess the iron distribution in 3D. 
Data acquisition was carried out on a probe corrected FEI Titan operated at $80 \mathrm{kV}$ acceleration voltage. The whole data set consisted of 48 SI ranging from $68.99^{\circ}$ to $64.74^{\circ}$ following a saxton scheme angle step with $55 \times 55$ spectra each, acquired during $0.1 \mathrm{~s}$. High angle annular dark field (HAADF) signal was acquired simultaneously. Afterwards, for data treatment, MVA methods were applied, namely principal component analysis (PCA) and ICA using EELSLab [12].

A whole set of EELS-SI tomograms contains an extremely large amount of spectra in comparison to conventional EELS experiments in 2D. Thus, despite the inconveniently original noisy data because of the reduced primary beam energy and exposure times used to prevent sample damage, those statistical approaches provide high quality results.

In the following we will describe the mathematical steps applied to the original signal to retrieve significant dataset of intensity $I$ distribution of the form $I(\mathrm{x}, \mathrm{y}, \theta)$, suitable as input data for the 3D tomographic reconstruction algorithm.

The first step of data treatment was merging the data together in EELSLab in order to obtain a greater SI onto which the statistical analysis could be applied. Therefore, the original four dimensional $(x, y, \theta, \Delta E)$ data set was transformed to $(x, y(\theta), \Delta E)$, the same format as a regular SI. After energy drift correction, PCA was applied on the data. This technique, which allows separating signals in a spectrum according to their variance [13], enhanced the signal-to-noise ratio by keeping only higher variance components. An example is shown in figure 2. From the noisy raw spectra obtained in the thinnest region (2b) and thickest region (2c) of the particle, enhanced $\mathrm{O}_{\mathrm{K}}, \mathrm{Fe}\left(\mathrm{L}_{3,2}\right)$ and $\mathrm{Co}\left(\mathrm{L}_{3,2}\right)$ edges were retrieved after PCA analysis (figures $2 \mathrm{e}$ and $2 \mathrm{f}$ ). Nevertheless, thickness effects were clearly noticed in figures $2 \mathrm{c}$ and $2 \mathrm{f}$ as revealed by the higher background contribution in the central part of the particle.

Further ICA was also carried out. This analysis consists on reducing the energy space, of a dimension given by the number of channels to a smaller space of orthogonal axes onto which every spectrum can be easily projected. The new basis axes (the independent components) are found according to the non-gaussian distribution (i.e. their independence from other signals) [14]. This analysis successfully retrieved the Fe oxide and Co oxide signals of the sample as well as the background signal before the oxygen $\mathrm{K}$ edge, which are three orthogonal axes of our new energy basis (figure 3a). Every single spectrum on the dataset can be represented as a function of the independent signals. Looking the other way round, every independent component has a scalar map for each spectrum image, which means that we have distribution maps for iron oxide, cobalt oxide and thickness. An example of these scalar maps at different angles are shown in figure $3 \mathrm{~b}$.

After PCA noise-reduction, the data were transformed back to the original four dimensions in order to proceed to traditional core loss quantification using the Egerton method [15]. Following background removal, edge intensities given by equation (1) were experimentally determined.

$$
I_{k}^{A}=N^{A} \cdot \sigma_{k}^{A} \cdot I_{T} \cdot \exp \left(-\frac{t}{\lambda}\right)
$$


where $I_{k}^{A}$ is the edge intensity of a $k$ transition for an element $A, N^{A}$ is the areal density of element $A, \sigma_{k}^{A}$ is the $k$ ionization cross-section of element $A, I_{T}$ the total transmitted beam intensity, $t$ is the sample thickness and $\lambda$ the ionization mean free path. The experimental results for the three extracted intensities for one projection are shown in figure 4 first column. The extracted $\mathrm{O}(\mathrm{K})$, Co $\left(\mathrm{L}_{32}\right)$ and $\mathrm{Fe}\left(\mathrm{L}_{32}\right)$ edge intensity maps (figures $4 \mathrm{a}, \mathrm{c}$ and $\mathrm{f}$ respectively) reveal a drop of intensity at the centre of the particle, again a clear indication of thickness effects. Plural scattering (convolution of the ionization edges with the low loss region) can be held responsible for the loss of intensity of these edges. The effects of plural scattering are avoided for thickness $(t)$ of the sample $t / \lambda \leq 0.3$ [15], where $\lambda$ is the inelastic scattering mean free path. The thickness of the particle is around $100 \mathrm{~nm}$ (as seen in figure 1) and the ionization mean free path for the extreme compositions (i.e. $\mathrm{Co}_{3} \mathrm{O}_{4}$ and $\mathrm{Fe}_{3} \mathrm{O}_{4}$ ), is of $73.5 \mathrm{~nm}$ and $74 \mathrm{~nm}$ respectively [15], which means that plural scattering is found for $t \geq 22 \mathrm{~nm}$. The experimental edge maps fail to fulfil the projection requirement [16] near the centre of the particle, where this drop in intensity is systematically found, but this problem is not found near the surface.

In a second step, quantification of $\mathrm{O}, \mathrm{Fe}$ and $\mathrm{Co}$ concentrations was extracted according to eq. 2 for three component quantification.

$A \%=\frac{N^{A}}{N^{A}+N^{B}+N^{C}}=\frac{I_{k}^{A} \cdot\left(\sigma_{k}^{A} \cdot I_{T} \cdot \exp \left(-\frac{t}{\lambda}\right)\right)^{-1}}{I_{k}^{A} \cdot\left(\sigma_{k}^{A} \cdot I_{T} \cdot \exp \left(-\frac{t}{\lambda}\right)\right)^{-1}+I_{k}^{B} \cdot\left(\sigma_{k}^{B} \cdot I_{T} \cdot \exp \left(-\frac{t}{\lambda}\right)\right)^{-1}+I_{k}^{C} \cdot\left(\sigma_{k}^{C} \cdot I_{T} \cdot \exp \left(-\frac{t}{\lambda}\right)\right)^{-1}} \approx \frac{I_{k}^{A} / \sigma_{k}^{A}}{I_{k}^{A} / \sigma_{k}^{A}+I_{k}^{B} / \sigma_{k}^{B}+I_{k}^{C} / \sigma_{k}^{C}}$

The results are shown in figure 4 second column. In these maps, the signals are only proportional to the areal densities and the ionization cross-sections, as the total intensity and the exponential factor cancel, if we consider that the mean free path through the sample does not effectively change, which is consistent with the previous calculations of mean free paths only changing $0.5 \mathrm{~nm}$ at most. Effectively, comparison of second and first column in figure 4 illustrates that quantification maps overcome the contrast inversion observed in edge maps. They fulfil the projection requirement, as the signal changes monotonically with a property of the sample, in this case elemental concentration, and it is not angle depending [10].

Combining the data of the ICA thickness maps and the quantification, a new kind of signal was extracted. As quantification images are directly proportional to the areal density of a given element and we have extracted a signal only proportional to thickness, both signals can be merged multiplying pixel by pixel the projection images. The intensity of this new signal can be also regarded as the contribution of a given element to the thickness found for every pixel, giving a signal proportional to the 
absolute number of atoms of that given element, density-thickness contrast images, which fulfil the projection requirement.

Spatial drift was measured on the Fe quantification using Imod [17] because of the higher signal to background ratio of this signal. Afterwards, these measures were used to correct all of the 9 datasets, which consisted on iron, cobalt and oxygen quantification, HAADF, sample thickness and iron oxide signal from ICA and the ICA thickness combined with core loss quantifications of $\mathrm{Fe}, \mathrm{Co}$ and $\mathrm{O}$. The same tilt axis correction, measured with Inspect3D software, was also applied. The latter software was used to reconstruct the tomograms, with 40 to 60 iterations of SIRT [18] in order to maximise the signal-to-background ratio.

\section{Results:}

In figure 5, the $\mathrm{Fe}$ signal is used to exemplify the information from an original projection, a voltex visualization of the reconstructed volume and a slice through that volume for the quantification maps (Fig.5 a-c), the ICA signals (Fig.5 d-f) and the density-thickness maps (Fig.5 g-i); showing the ability of those signals to reconstruct chemical information in 3D. Moreover, a visualization of the reconstructed volume for the HAADF signal is shown (Fig.5j), as well as a comparison between the quantification reconstructions of $\mathrm{Fe}$ and $\mathrm{Co}$ (Fig.5k) and the superposition of HAADF and thickness maps from ICA (Fig.51). The comparison of iron and cobalt is extended in figure 6 , where orthoslices of density-thickness reconstructions for both elements are shown.

\section{Discussion:}

Interestingly, all the chosen signals could be properly reconstructed, leading to volumes containing chemical information. It must be taken into account that those signals suffering from thickness effects show lower intensities in the center of the reconstruction, but that have no effect on the shape information. The reconstruction of the HAADF STEM tomography is the most well established 3D reconstruction technique in the TEM and thus the HAADF signal co-acquired during the tilt series will be used for assessment of the quality of the other reconstructions.

Regarding chemical information, an interesting result was revealed: the comparison between iron and cobalt signals showed that some of the iron which was intended to penetrate the structure remains instead on the outer surface. Moreover, the rest of the iron content is uniformly distributed inside the particle, as seen in the density-thickness maps reconstruction (Fig.5 g-i).

We can observe that the oxygen and cobalt reconstructions for extracted signal and quantification (not shown) are coincident with HAADF volume. This was expected, as cobalt oxide formed the original mesoporous structure. Conversely, iron signals reconstruct volumes greater than the HAADF. The particles are richer in iron at the border and therefore, iron related chemical signals give a sharp interface between the particle and the background, especially quantification and density-thickness maps, where HAADF signal is very low and has fallen to background levels due to the small thickness. These results show that iron signals reconstruct more precisely the edge of the particles than HAADF. On the other hand, the thickness signal has the drawback of underestimating the border more than the HAADF signal, as observed in the original 
projections. This is due to the statistical nature of the signal, which assigns it a low contribution to the spectra at the border. However, the most interesting feature of this signal is that it is insensitive to the chemistry of our sample and independent of multiple scattering, a characteristic not found in any other signal used for electron tomography.

\section{Conclusions:}

As a conclusion, EELS SI tomography is shown to be able to reconstruct chemical information of a sample in three dimensions. Moreover, the application of MVA to the data opens a new range of applications, reducing the limitations due to beam sensitive materials or samples with components with overlapping edges, where core-loss extraction using background estimation fails. This has been illustrated on mesoporous $\mathrm{Co}_{3} \mathrm{O}_{4}$ particles filled with $\mathrm{Fe}_{\mathrm{x}} \mathrm{Co}_{(3-\mathrm{x})} \mathrm{O}_{4}$.

\section{Acknowledgements:}

\section{Keywords:}

Tomography, EELS, core-loss, MVA

\section{Highlights:}

- EELS-SI tomography was performed at low voltage and low acquisition times.

- MVA has been applied for noise reduction and information extraction.

- Tomographic reconstruction has been achieved for chemical information.

- Elemental distribution extraction in 3D has been proved. 

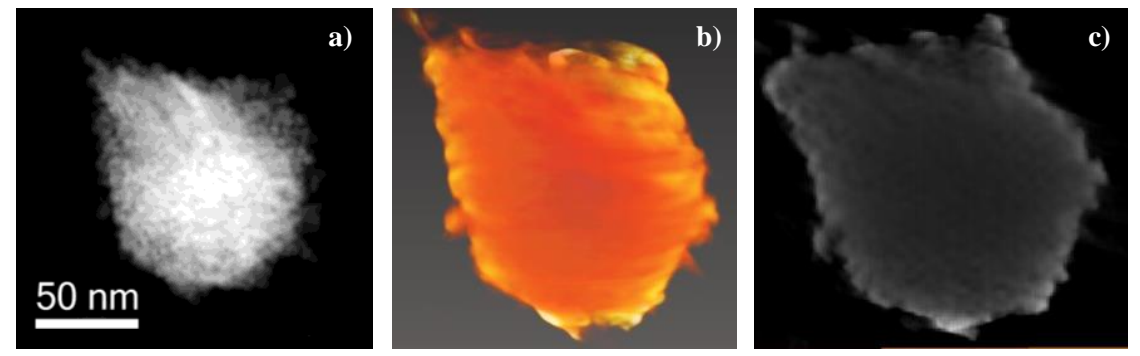

Figure 1: a) HAADF projection of the particle used in the analysis. b) Reconstruction using the HAADF projections and $\mathbf{c}$ orthoslice through the volume of the particle. Note that this HAADF signal is not the one acquired with the SI. 

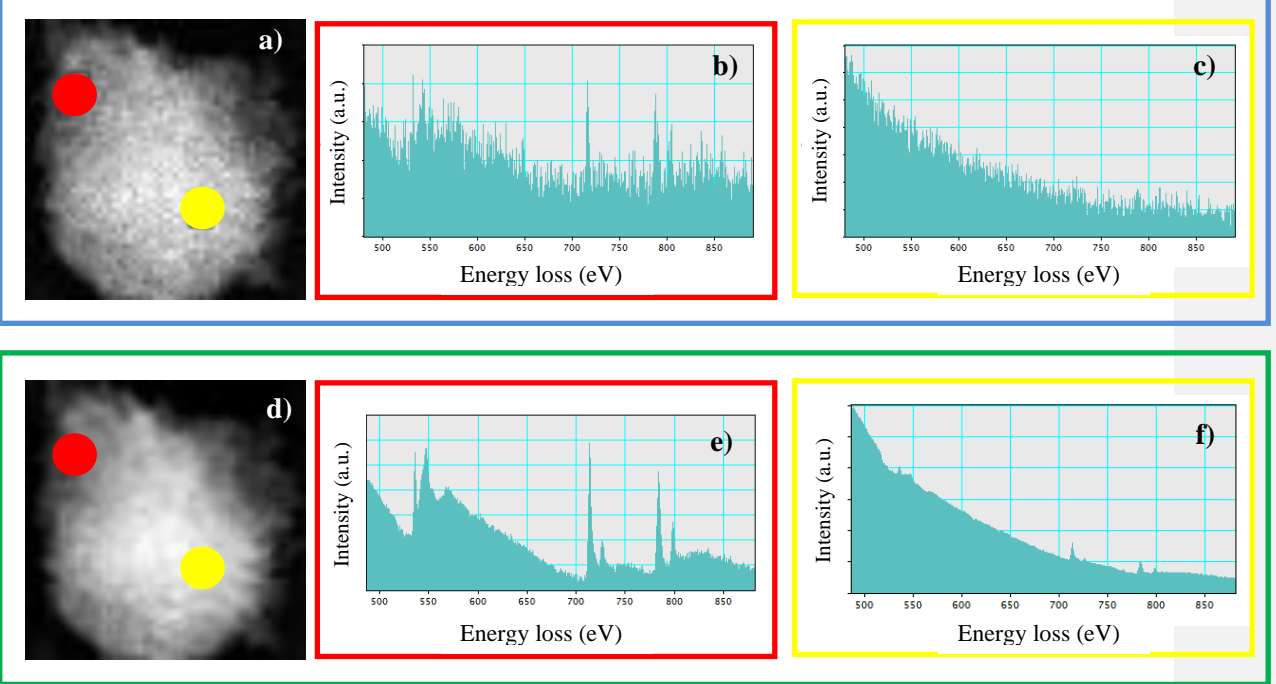

Figure 2: Spectrum images at $68.99^{\circ}$ before (framed in blue) and after (framed in green) PCA assisted noise reduction. Sample spectra from the border of the particle ( $\mathbf{b}$ and $\mathbf{e}$, in red) and the thicker part (c and $\mathbf{f}$, in yellow) are shown. 


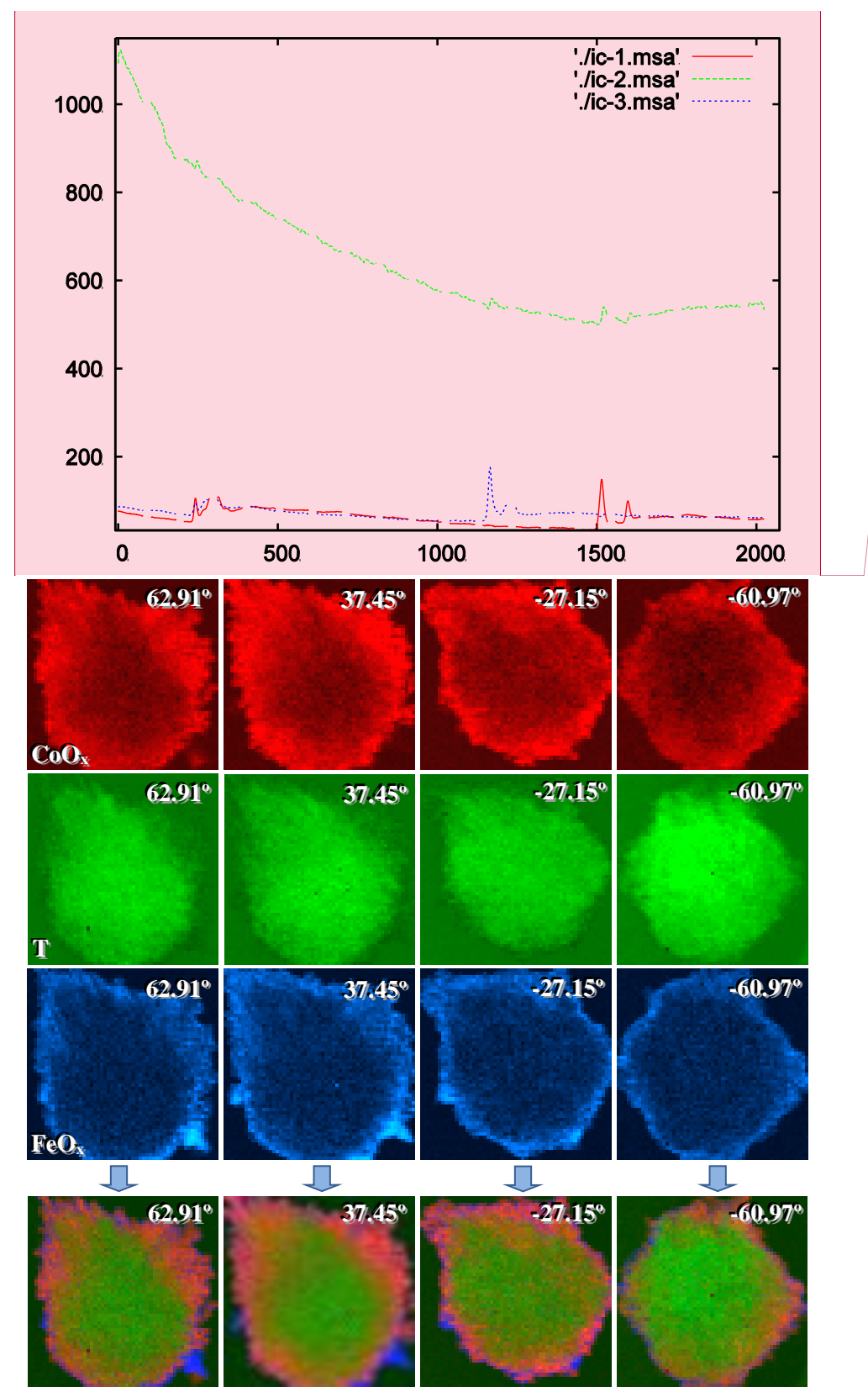

Figure 3: Independent components (unity vectors of our new basis) and extracted IC maps for four different projections: cobalt oxide $\left(\mathrm{CoO}_{\mathrm{x}}\right)$ in red, thickness $(\mathrm{T})$ in green and iron oxide $\left(\mathrm{FeO}_{\mathrm{x}}\right)$ in blue. RGB composites are given for the four tilt angles in order to compare the spatial distribution and magnitude of each component. 

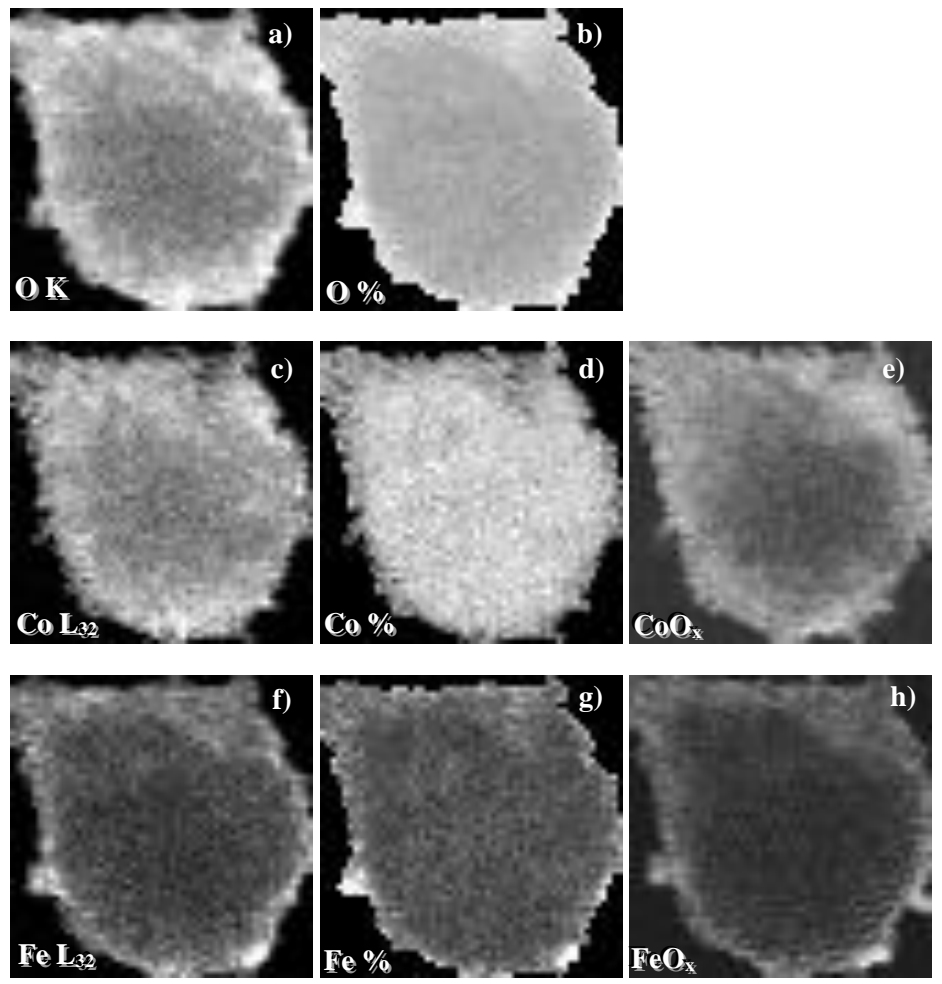

Figure 4: First column: Edge intensity maps for Oxigen $\mathrm{K}(\mathbf{a})$, Cobalt $\mathrm{L}_{32}(\mathbf{c})$ and $\operatorname{Iron} \mathrm{L}_{32}(\mathbf{e})$ calculated from equation 1 after noise reduction. Second column: Elemental quantification maps for $\mathrm{O}(\mathbf{b}), \mathrm{Co}(\mathbf{d})$ and $\mathrm{Fe}(\mathbf{f})$ from equation 2. Third column: Cobalt oxide (g) and iron oxide (h) maps extracted from ICA analysis. Note that the quantification maps overcome the intensity inversion at the centre of the particles. Remark also the similarities between the independent components and their respective metal map. 

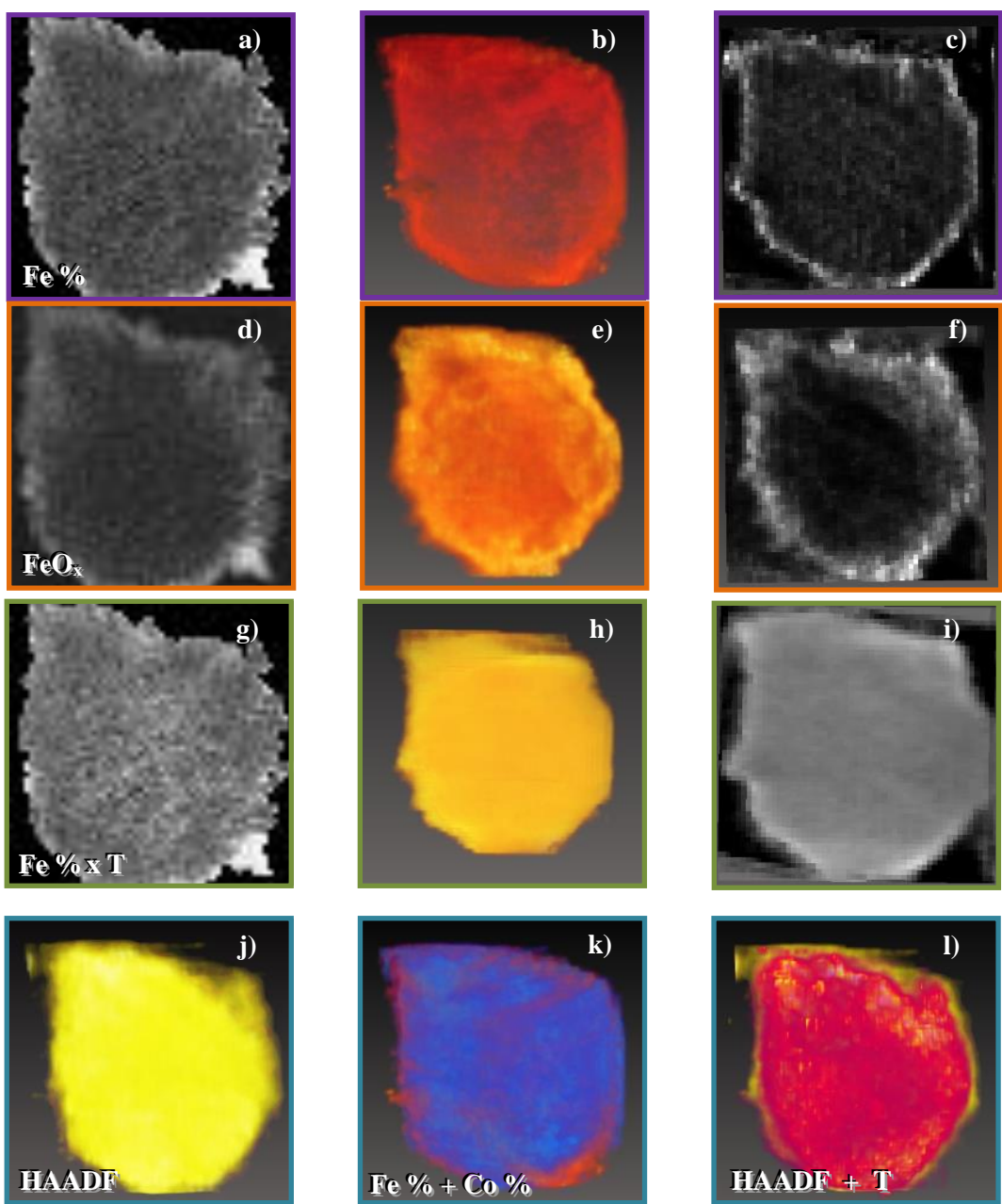

Figure 5: a) iron map at $68.99^{\circ}$ from Egerton quantification, b) voltex visualization of the reconstruction of iron maps and c) slice through the reconstructed volume. d) Iron oxide map from ICA, e) voltex visualization of the reconstruction of ICA iron oxide maps and f) slice through the reconstructed volume. g) Iron density-thickness map from ICA, h) voltex visualization of the reconstruction of ICA thickness maps related to iron quantification and i) slice through the reconstructed volume. j) HAADF reconstruction from the co-acquired signal. k) Superposition of the voltex visualizations of $\mathrm{Co}$ (blue) and $\mathrm{Fe}$ (orange) obtained from quantification and l) HAADF (yellow) and thickness maps from ICA (violet). 

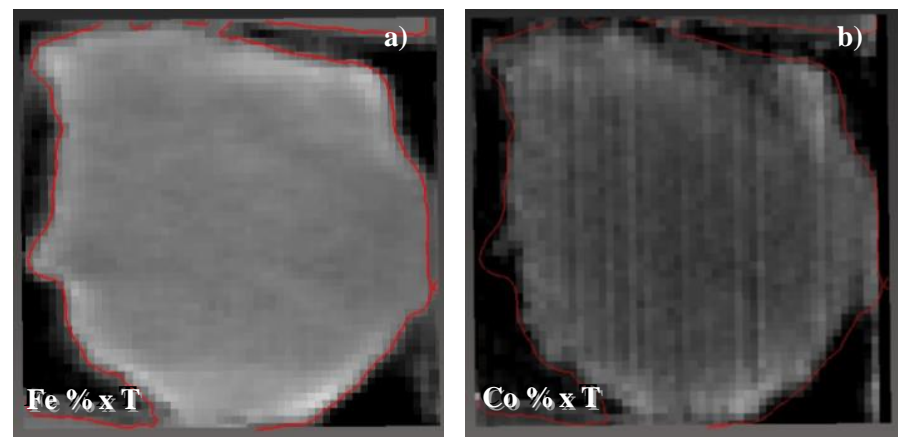

Figure 6: a) Orthoslice through the volume of the reconstructed iron density-thickness map with a red isoline at the border. b) Orthoslice through the volume of the reconstructed cobalt density-thickness map with the same isoline. It can be observed that the iron has higher intensity than the cobalt at the edges of the particle for the same slice. 
Reference List

[1] K. Jarausch, P. Thomas, D.N. Leonard, R. Twesten, C.R. Booth, Four-dimensional STEM-EELS: Enabling nano-scale chemical tomography, Ultramicroscopy. 109 (2009) 326-337.

[2] G. Möbus, R.C. Doole, B.J. Inkson, Spectroscopic electron tomography, Ultramicroscopy. 96 (2003) 433-451.

[3] M. Weyland, P.A. Midgley, Extending Energy-Filtered Transmission Electron Microscopy (EFTEM) into Three Dimensions Using Electron Tomography, Microscopy and Microanalysis. 9 (2003) 542.

[4] R.D. Leapman, E. Kocsis, G. Zhang, T.L. Talbot, P. Laquerriere, Three-dimensional distributions of elements in biological samples by energy-filtered electron tomography, Ultramicroscopy. 100 (2004) 115-125.

[5] M. Varela, M.P. Oxley, K.G. Roberts, J. Garcia-Barriocanal, A.R. Lupini, S.N. Rashkeev, C. Leon, K.M. Krishnan, J. Santamaria, S.T. Pantelides, S.J. Pennycook, Spectroscopic imaging of oxide interfaces with aberration corrected probes RID A-5587-2008, Microsc. Microanal. 13 (2007) 142-143.

[6] M. Bosman, M. Watanabe, D.T.L. Alexander, V.J. Keast, Mapping chemical and bonding information using multivariate analysis of electron energy-loss spectrum images, Ultramicroscopy. 106 (2006) 1024-1032.

[7] T. Yamazaki, Y. Kotaka, Y. Kataoka, Analysis of EEL spectrum of low-loss region using the Cs corrected STEM-EELS method and multivariate analysis, Ultramicroscopy. 111 (2011) 303-308.

[8] M. Weyland, P.A. Midgley, J.M. Thomas, Electron Tomography of Nanoparticle Catalysts on Porous Supports:â€\%॰ A New Technique Based on Rutherford Scattering, The Journal of Physical Chemistry B. 105 (2001) 7882-7886.

[9] G. Möbus, B.J. Inkson, Three-dimensional reconstruction of buried nanoparticles by element-sensitive tomography based on inelastically scattered electrons, Appl. Phys. Lett. 79 (2001) 1369-1371.

[10] P.A. Midgley, M. Weyland, 3D electron microscopy in the physical sciences: the development of Z-contrast and EFTEM tomography, Ultramicroscopy. 96 (2003) 413-431.

[11] I. Arslan, T.J.V. Yates, N.D. Browning, P.A. Midgley, Embedded Nanostructures Revealed in Three Dimensions, Science. 309 (2005) 2195-2198.

[12] F. de la Peña, M.-. Berger, J.-. Hochepied, F. Dynys, O. Stephan, M. Walls, Mapping titanium and tin oxide phases using EELS: An application of independent component analysis, Ultramicroscopy. 111 (2011) 169-176.

[13] R.N. Cochran, F.H. Horne, Statistically weighted principal component analysis of rapid scanning wavelength kinetics experiments, Anal. Chem. 49 (1977) 846-853. 
[14] N. Bonnet, D. Nuzillard, Independent component analysis: A new possibility for analysing series of electron energy loss spectra, Ultramicroscopy. 102 (2005) 327-337.

[15] Egerton R. F., Electron Energy-Loss Spectroscopy in the Electron Microscope, Second ed., Plenum Press, New York, 1996.

[16] P.W. Hawkes, The Electron Microscope as a Structure Projector, (2006) 83-111.

[17] J.R. Kremer, D.N. Mastronarde, J.R. McIntosh, Computer visualization of threedimensional image data using IMOD, J. Struct. Biol. 116 (1996) 71-76.

[18] P. Gilbert, Iterative methods for the three-dimensional reconstruction of an object from projections, J. Theor. Biol. 36 (1972) 105-117. 